\title{
Aerodynamic design, analysis, and validation techniques for the Tianwen-1 entry module
}

\author{
Qi Li $^{1}(\bowtie)$, Wei Rao ${ }^{1}$, Xiaoli Cheng ${ }^{2}$, Haogong Wei ${ }^{1}$, Chuang Wang ${ }^{1}$, and Jie Dong ${ }^{1}$ \\ 1. Beijing Space Vehicle Design Department, China Academy of Space Technology, Beijing 100094, China \\ 2. China Academy of Aerospace and Aerodynamics, Beijing 100074, China
}

\begin{abstract}
The clear differences between the atmosphere of Mars and the Earth coupled with the lack of a domestic research basis were significant challenges for the aerodynamic prediction and verification of Tianwen-1. In addition, the Mars entry, descent, and landing (EDL) mission led to specific requirements for the accuracy of the aerodynamic deceleration performance, stability, aerothermal heating, and various complex aerodynamic coupling problems of the entry module. This study analyzes the key and difficult aerodynamic and aerothermodynamic problems related to the Mars EDL process. Then, the study process and results of the design and optimization of the entry module configuration are presented along with the calculations and experiments used to obtain the aerodynamic and aerothermodynamic characteristics in the Martian atmosphere. In addition, the simulation and verification of the low-frequency free oscillation characteristics under a large separation flow are described, and some special aerodynamic coupling problems such as the aeroelastic buffeting response of the trim tab are discussed. Finally, the atmospheric parameters and aerodynamic characteristics obtained from the flight data of the Tianwen-1 entry module are compared with the design data. The data obtained from the aerodynamic design, analysis, and verification of the Tianwen-1 entry module all meet the engineering requirements. In particular, the flight data results for the atmospheric parameters, trim angles of attack, and trim axial forces are within the envelopes of the prediction deviation zones.
\end{abstract}

\section{KEYWORDS}

Mars entry, descent, and landing (EDL)

design and optimization of the entry module configuration

calculations and experiments of aerodynamic and aerothermodynamic characteristics

low-frequency free oscillation under large separation flow special aerodynamic coupling problems

flight data

\section{Research Article}

Received: 16 September 2021

Accepted: 1 November 2021

(C) The Author(s) 2022

\section{Introduction}

On May 15, 2021, the Tianwen-1 entry module entered the Martian atmosphere in a semiballistic manner, underwent a process of aerodynamic deceleration, parachute deceleration, and dynamic backpush deceleration, and successfully landed at the preselected landing zone in the southern part of the Utopia Plain on Mars. China has thus become the second country (after the United States) to perform a successful entry, descent, and landing (EDL) mission on Mars.

The exploration of Mars dates back to the 1960s [1]. To date, China, the United States, Japan, Russia, Europe, India, and other countries and regions have launched nearly 50 Mars exploration missions, less than half of which have been fully successful. For missions to the surface of Mars, only 10 probes from China and the United States have succeeded.

Compared with the Earth reentry, the Mars EDL process has different characteristics and difficulties in the aerodynamic design of the entry module, which are reflected in the main aspects described below.

\subsection{Aerodynamic configuration design and optimization for rarefied atmosphere entry}

The density of the Martian atmosphere is only $1 \%-10 \%$ of that on the Earth at the same height $[2,3]$. Therefore, the Mars entry module needs an aerodynamic configuration 


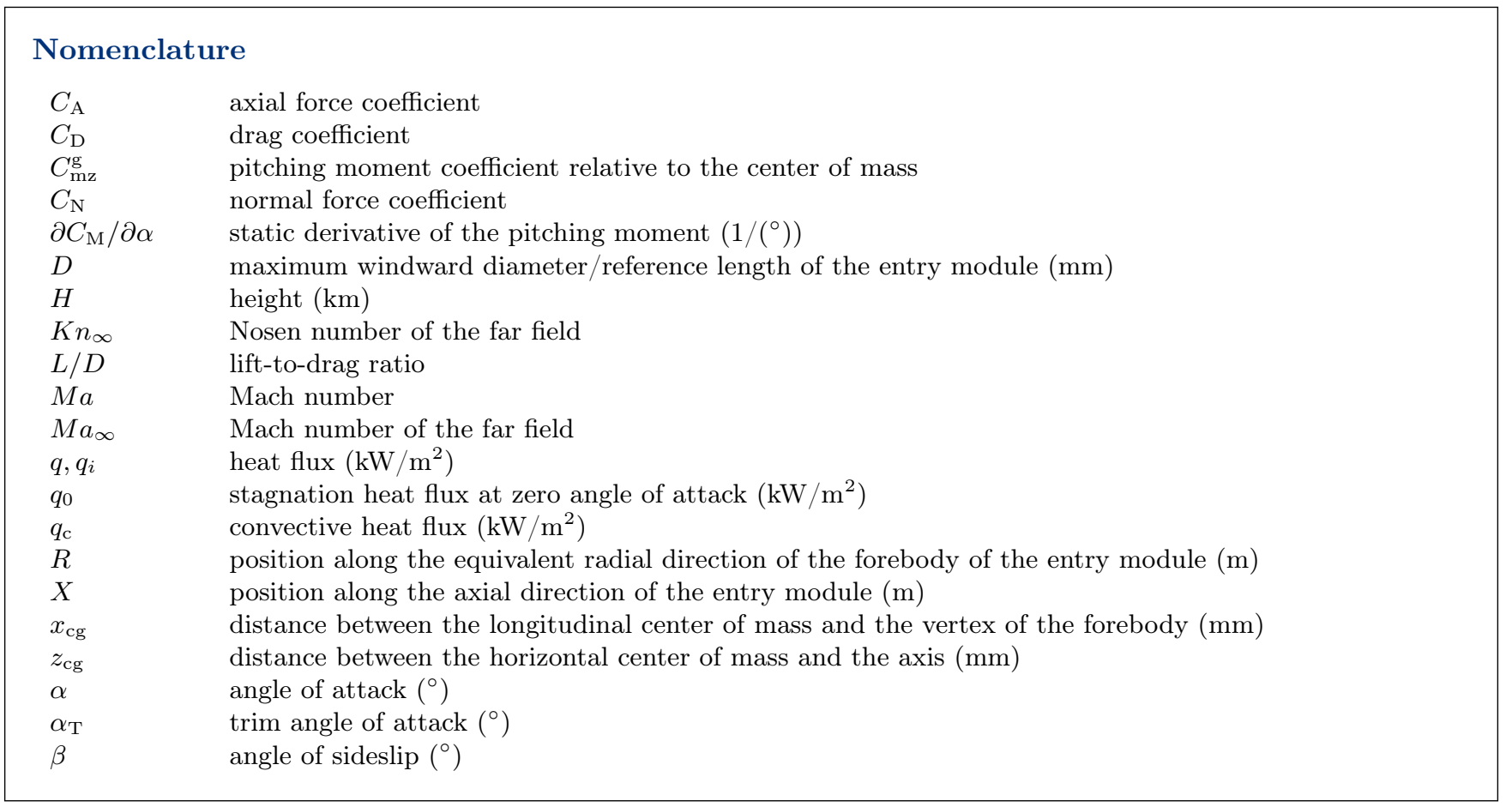

with a high deceleration performance compared to that required for the Earth reentry. In addition, to maximize the landing payload ratio, the thermal protection system for the entry module should have the characteristics of an ultralow ablative and ultralight structure. All these factors lead to specific requirements for the configuration of a Mars entry module [4]. Before Tianwen-1, the successful entry modules of the United States Mars landers all had a configuration with a large blunt cone forebody and short inverted cone afterbody [5-7]. The deceleration performance and lightweight structure performance of the configuration of China's previous recoverable satellites [8], the Shenzhou spaceship [9], and Chang'e-5 return capsule [10], with a mature technical basis cannot meet the requirements of a Mars EDL mission. In summary, China's Mars exploration mission must develop a new aerodynamic configuration design for the entry module, which is technically difficult.

\subsection{Aerodynamic and thermodynamic prediction and verification techniques applicable to the Martian atmosphere}

The atmosphere of Mars is composed of $95.7 \% \mathrm{CO}_{2}$, $2.7 \% \mathrm{~N}_{2}, 1.6 \% \mathrm{Ar}$, and other trace molecules [3]. The $\mathrm{CO}_{2}$ environment of the Martian atmosphere is more easily dissociated, ionized, and excited by vibration.
As a result, the aerodynamic and thermodynamic characteristics of a Mars entry module are strongly affected by the occurrence of chemical reactions in a high-temperature atmosphere [11]. In addition, the low density of the Martian atmosphere causes a more severe rarefied effect during the Mars entry than that during the Earth reentry [12]. However, calculation methods based on the effects of the high-temperature thermochemical non-equilibrium, rarefied gas, and other complex factors of the Martian atmospheric composition have not been established in China. Meanwhile, the original aerodynamic and thermodynamic testing techniques based on air media cannot be directly applied to the verification of aerodynamic/thermodynamic characteristics in the Martian atmosphere. Research on suitable test equipment and testing techniques must be conducted.

To solve the above problems, the Tianwen- 1 pneumatic design team and the domestic pneumatic test site jointly completed a study consisting of the selection and optimization of the entry module configuration along with analysis and verification of its aerodynamic and aerothermodynamic characteristics. Combining engineering calculations, numerical simulations, and ground tests, the pneumatic team determined an aerodynamic configuration suitable for entry deceleration 
in the rarefied Martian atmosphere and obtained the aerodynamic characteristics and aerothermal environments of the Mars entry module. This paper presents the main concepts of the aerodynamic design, analysis, and verification of the Tianwen-1 entry module, as well as the research process and the major results in each research area. Finally, a comparison between the aerodynamic design data of the entry module and flight inversion data is provided.

\section{Selection and optimization of the entry module configuration}

\subsection{Selection of the configuration design}

The high reliability, high landing accuracy, and high load ratio requirements of a Mars EDL mission lead to high demands on the lift and drag performance, static/dynamic stability, thermodynamic environment, and effective volume ratio of the entry module. After carrying out an EDL ballistic attitude simulation, assembly layout, and quality budget based on the overall task index, the maximum size envelope, layout space constraints, resistance performance requirements, thermal environment constraints, stability, and interface size constraints can be determined, which are the main factors for selecting the configuration of the module.

Based on research and engineering experience, typical semiballistic reentry and entry modules use large blunt wind-facing forebodies and inverted cone afterbodies connected with a small-radius arc [13, 14]. The initial design selection is based on forebody shapes of a ball crown and ball cone as shown in Table 1 [15]. The selection steps are as follows.

(1) The pneumatic design variables are established and the target function is determined, including the lift and drag coefficients, static stability, and surface heat flow distribution. It is required that the drag coefficient is at least 1.5, the lift-to-drag ratio is at least 0.12 , the static stability derivative is not more than -0.001 , and the maximum laminar heat flow is not higher than $300 \mathrm{~kW} / \mathrm{m}^{2}$.

(2) Using a multi-objective optimization method based on a random drop point/ray, alternative entry module forebody shapes are obtained based on a ball crown and ball cone. The corresponding aerodynamic characteristic data used in the multi-objective optimization are then obtained based on the modified Newton flow method with an equivalent ratio heat ratio model [16].

The static aerodynamic characteristics of the two alternative configurations at hypersonic speeds are numerically simulated using the Navier-Stokes (N-S) equation based on the equivalent specific heat ratio model, and the results are compared in Fig. 1. It can be seen that under the same Mach number and angle of attack, the drag coefficient and lift-to-drag ratio of the ball-cone forebody configuration are higher than those of the ball-crown forebody configuration.

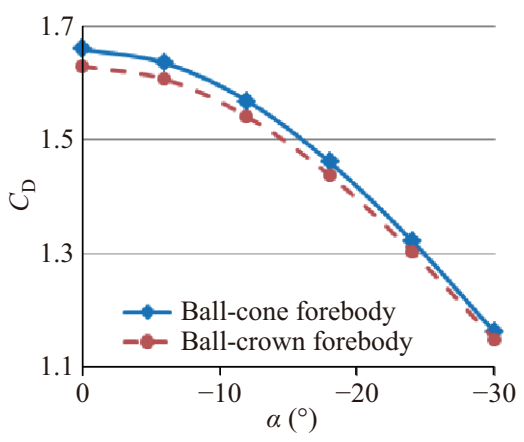

(a) Drag coefficient

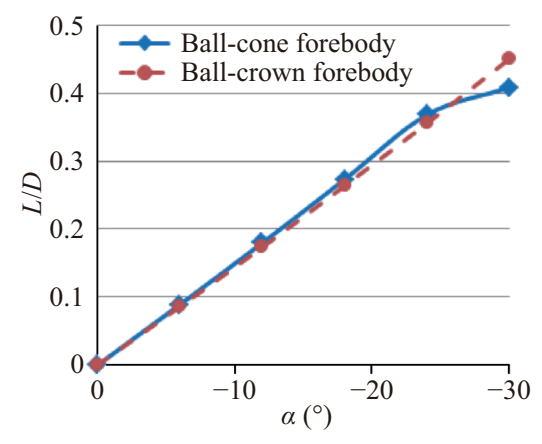

(b) Lift-to-drag ratio

Fig. 1 Comparison of the lift and drag characteristics of the ball-cone and ball-crown forebody configurations $(M a=10)$.

Figure 2 compares the differences in the relationship between the center position and the contours of the trim angle of attack and the static derivative of the pitching moment for the ball-cone forebody and ball-crown forebody configurations [16]. The trim line represents the characteristic line with zero pitching moment at the centroid, and the sta-isoline represents the characteristic line with a zero static derivative of the pitching moment. In addition, "cg" indicates the center of mass of the entry module, $x_{\mathrm{cg}} / D$ represents the longitudinal centroid 
Table 1 Alternative configurations of the Mars entry module

Category of the forebody shape
Configuration diagram
Maximum windward diameter, $D(\mathrm{~mm})$
Total length, $L$ (mm)
Bluntness of the spherical crown, $R_{\mathrm{N}} / D$
Windward half-cone angle, $\theta_{1}\left(^{\circ}\right)$

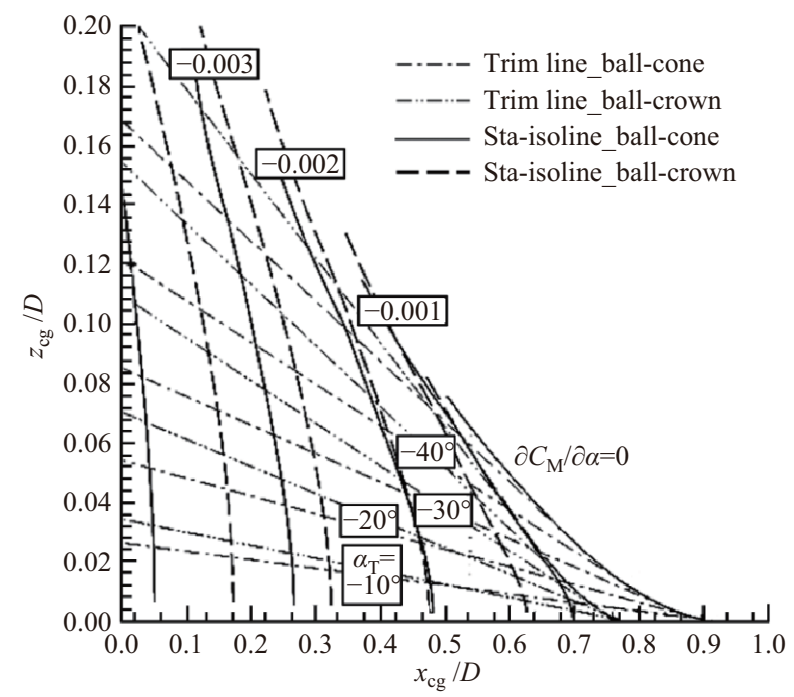

Fig. 2 Trim line and the stability isoline distribution in the CG plane for the ball-cone and ball-crown forebody configurations.

relative to the windward diameter of the cabin, i.e., the reference length, and $z_{\mathrm{cg}} / D$ represents the value of the transverse centroid relative to the reference length. It can be seen that in the same overall size envelope, for the same lift-to-drag ratio requirements, the ball-cone forebody configuration requires a smaller horizontal centrifugal offset, which is more conducive to the layout of the bulkhead equipment. Both configurations are pitched gently and steadily over a wide range of centers of mass. The static stability margin of the ball-cone forebody configuration is slightly weaker than that of the ball-crown forebody configuration. However, the pitch stability of the ball-cone forebody configuration is less sensitive to the longitudinal center of mass, which is conducive to the design of the assembly layout.

The N-S equation numerical simulation method based on the thermochemical non-equilibrium and laminar flow model of the Mars atmosphere is used to calculate the surface heat flux distributions at the peak time of the heat flux for the ball-cone and the ball-crown forebody configurations, as shown in Fig. 3. As shown in the figure, the maximum heat flux of the surface of both shapes does not exceed $250 \mathrm{~kW} / \mathrm{m}^{2}$, and the heat flux of the ball-crown forebody is lower than that of the ball-cone forebody. The thermal environments of the two configurations are basically the same over the shoulder.

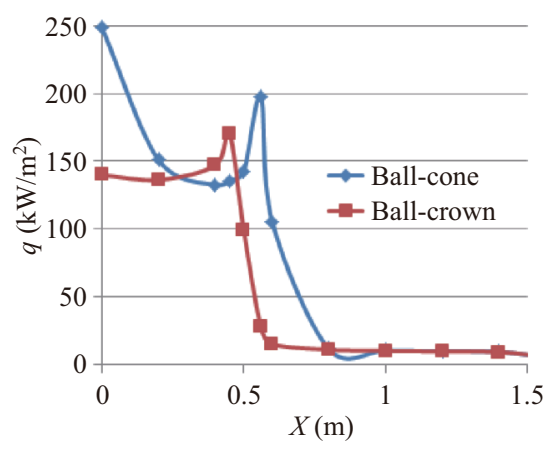

Fig. 3 Peak heat flux curves under the nominal trajectory for the ball-cone and ball-crown forebody configurations $(\alpha=$ $\left.-10^{\circ}\right)$.

In summary, after comparing and analyzing the lift and drag performance, flat layout performance, static stability, and aerothermal heating environment of the ball-cone and ball-crown forebody configurations, the 
ball-cone forebody configuration was selected as the basic configuration of the Tianwen-1 entry module.

\subsection{Design of the trim angle of attack reco- very system: trim tab layout scheme}

To achieve controlled Mars entry deceleration, the lateral offset of the center of mass is used to obtain a certain trim angle of attack; thus, the entry module uses the guidance navigation control (GNC) system to stabilize the trajectory and attitude during the main pneumatic deceleration. However, owing to the large blunt forebody and short blunt afterbody, the dynamic stability is poor in transonic and supersonic regions. As a result, attitude oscillations combined with the trim angle of attack may cause the total angle of attack of the entry module to exceed the indicated constraints for safe parachute deployment. To reduce the impact of posture oscillation, it is possible to return the trim angle of attack of the entry module to near $0^{\circ}$ before parachute deployment. For this purpose, the Mars Science Laboratory (MSL) ejected a mass to restore the center of mass of the module to the axis of revolution before deploying the parachute, but the mass cost of this method was large (approximately $150 \mathrm{~kg}$ ) [17]. To minimize the structural and counterweight mass of the spacecraft system under tight mass constraints, the aerodynamic team innovatively proposed a deployable trim tab layout, i.e., installation of a tab on the windward sidewall of the entry module that unfolds before the parachute is deployed. Thus, the aerodynamic moment caused by the flow around the tab changes the trim angle of attack of the module. After several rounds of pneumatic iteration, the size parameters and layout position of the tab to return the trim angle of attack of the module to $0^{\circ}$ are finally determined. As a result, the structure and mechanism design confirm that the weight cost of the trim wing is only $15 \mathrm{~kg}$. Figure 4 compares the angle of attack recovery systems of the Tianwen-1 and MSL entry modules.

\subsection{Optimization of the afterbody configu- ration}

To solve the problems of weight gain, a low effective volume ratio, and poor transonic and supersonic dynamic stability of the three-cone afterbody configuration of the entry module, the aerodynamic team optimized the configuration. First, several connection schemes of the afterbody structure that could reduce the system quality were proposed in the structural subsystem, and the aerodynamic configuration design was carried out by the pneumatic team. Then, the aerodynamic team compared the lift-drag performance and static and dynamic stability of the adaptively optimized afterbody

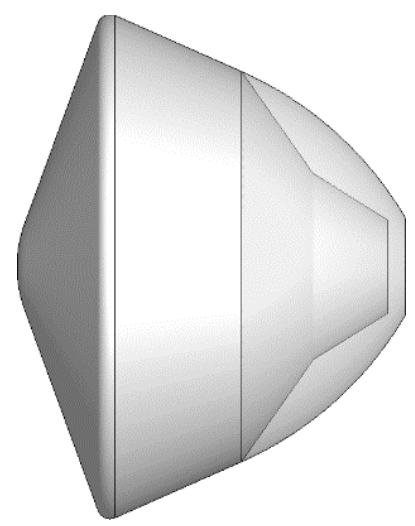

Fig. 5 Schematic diagram of the optimized shape of the module.

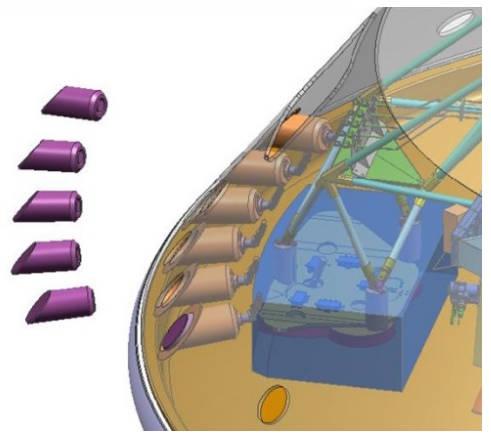

(a) Entry balance mass ejection (SUFR) for the MSL entry module

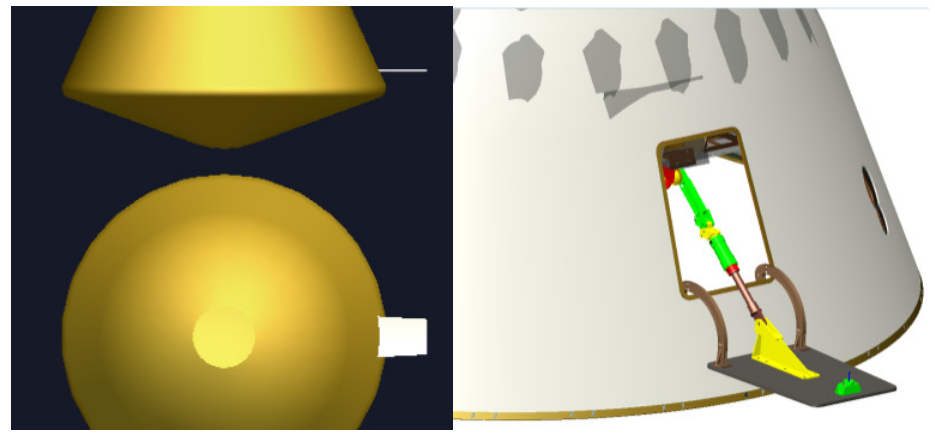

(b) Deployable trim tab for the Tianwen-1 entry module

Fig. 4 Comparison of the angle of attack recovery systems of the Tianwen-1 and MSL entry modules. 
Table 2 Comparison of parameters before and after optimization of the afterbody

Parameter Configuration category

\begin{tabular}{|c|c|c|}
\hline 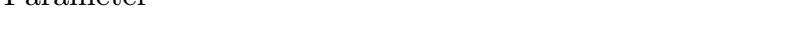 & Three-cone afterbody & Ball-cone afterbody \\
\hline Surface area of the afterbody $\left(\mathrm{m}^{-2}\right)$ & 16.169 & 17.381 \\
\hline Volume of the afterbody $\left(\mathrm{m}^{-3}\right)$ & 7.815 & 9.099 \\
\hline Volume ratio of the afterbody, $k$ & 0.483 & 0.524 \\
\hline Maximum dynamic derivative $\left(M a=1.2, \alpha=-4^{\circ}-4^{\circ}\right)$ & 1.130 & 1.040 \\
\hline Maximum dynamic derivative $\left(M a=1.5, \alpha=-4^{\circ}-4^{\circ}\right)$ & 1.310 & 1.250 \\
\hline
\end{tabular}

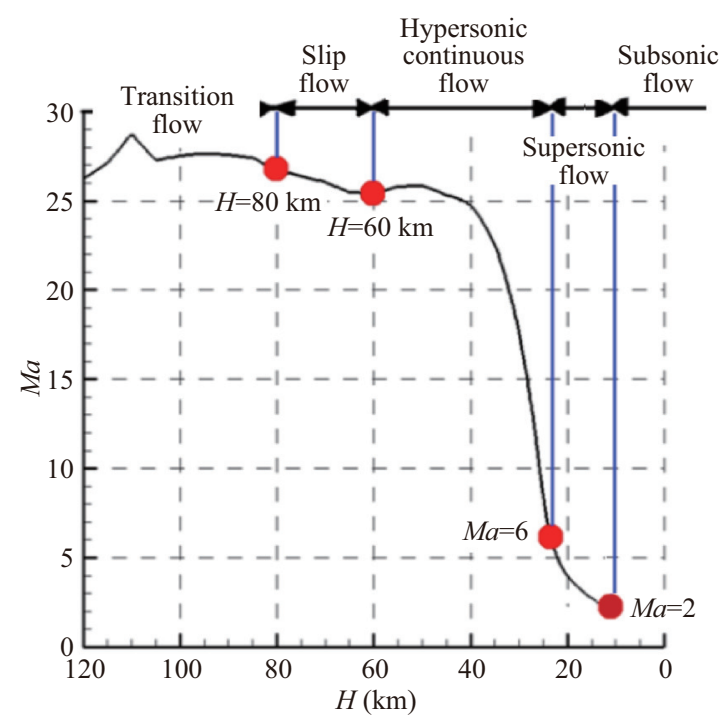

(a) Division of flow and velocity regions in the process of pneumatic deceleration

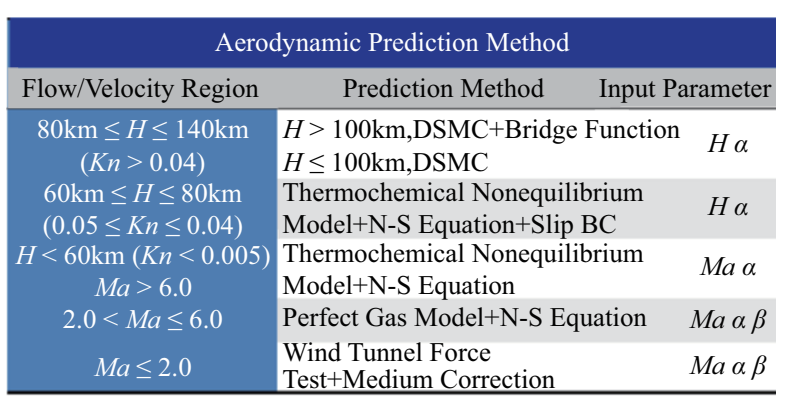

(b) Aerodynamic prediction algorithm

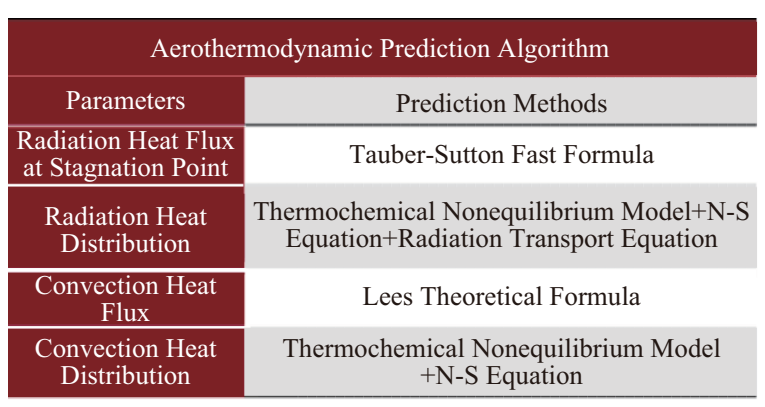

(c) Aerothermodynamic prediction algorithm

Fig. 6 Main research routes for the aerodynamic and aerothermodynamic characteristics of the Tianwen-1 entry module.

shapes: the afterbody with two cones, the afterbody with a single cone, and the afterbody with a ball cone as shown in Fig. 5. Finally, the ball-cone afterbody was determined as the optimal shape with the best comprehensive aerodynamic performance and the lightest system mass. Thus, the weight of the frame structure is effectively reduced, the layout space of the entry module is increased, and the oscillation in the angle of attack under transonic and supersonic speeds is reduced.

\section{Prediction and verification of the aero- dynamic and aerothermodynamic char- acteristics of the Mars entry module}

\subsection{Main research route}

Based on the definition of compressible and rarefied transition flow along with the research experience of similar detectors abroad [18], the entry pneumatic deceleration process of the Tianwen-1 entry module can 
be divided into a transition flow region, slip flow region, hypersonic continuous flow region, supersonic region, and subsonic region. Based on the characteristics of the flow field around the module in the Martian atmosphere and the existing algorithms, methods for predicting the aerodynamic force and aerothermal environment suitable for each flow or velocity region are determined, and algorithm research is carried out. Figure 6 shows the main research routes for the aerodynamic and aerothermodynamic characteristics of the Tianwen-1 entry module.

\subsection{Pneumatic algorithms for the real gas effect at high temperatures in the Martian atmosphere}

The main medium for the free flow of a Mars entry

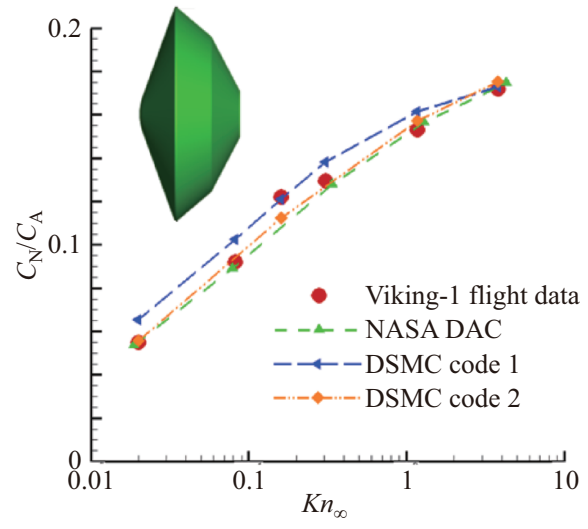

(a) Ratios of the axial and normal force coefficients in the transition flow region (Viking-1)

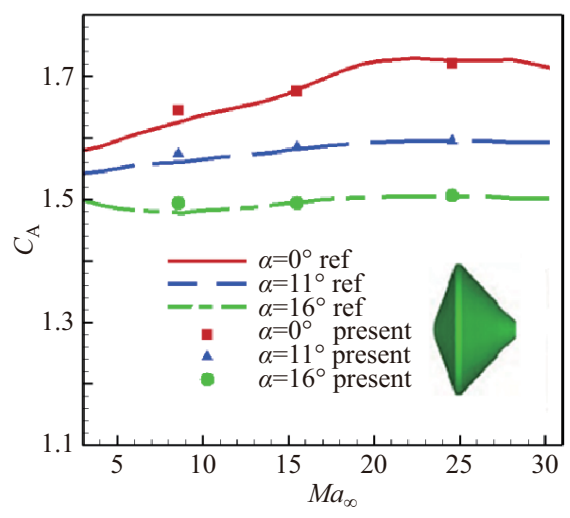

(c) Axial force coefficients in a hypersonic continuous flow (Phoenix) module is the flow of $\mathrm{CO}_{2}$. As a triatomic molecule, the thermal freedom of $\mathrm{CO}_{2}$ is greater than that of air, and the molecular vibrational energy excitation characteristics are stronger than those of air and far more complex than those of diatomic or monatomic molecules. In addition, hypersonic flow in the Martian atmosphere is accompanied by shock layer surface catalytic dissociation, ionization, chemical non-equilibrium thermodynamics, and the ablation effect of real gas. These complex effects have a significant impact on the shock, pressure, and temperature fields of the module, which may change the overall aerodynamic force and the distribution features of the aerothermal environment [19].

To predict the Martian atmosphere hypersonic aerodynamic and aerothermodynamic characteristics of the Tianwen-1 entry module accurately, the research

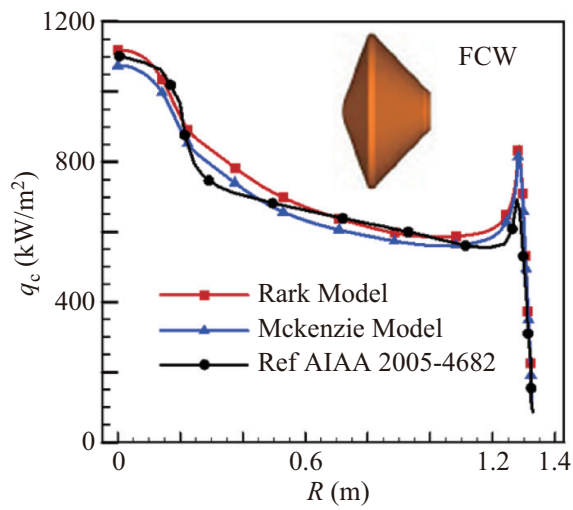

(b) Heat flux distribution of the surface (MPF)

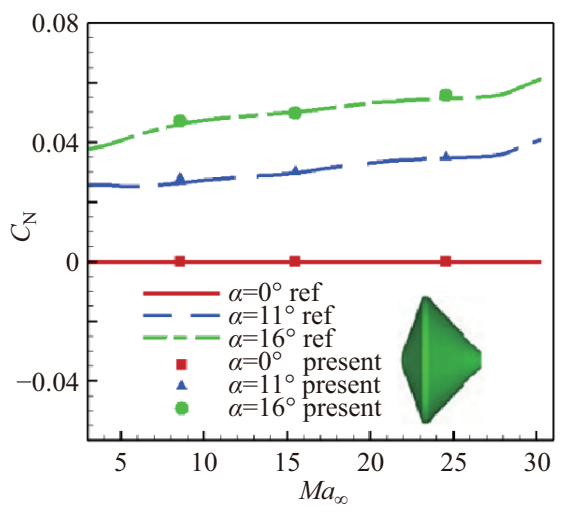

(d) Normal force coefficients in a hypersonic continuous flow (Phoenix)

Fig. 7 Aerodynamic and thermodynamic parameters obtained using the calculation method in this study compared with reference data. 
team carried out studies on the equivalent specific heat ratio model of the Martian atmosphere [20], chemical reaction models, thermodynamic models, and boundary conditions of the Martian atmosphere based on the chemical non-equilibrium N-S equation [21-23] and the direct simulation Monte Carlo (DSMC) aerodynamic simulation algorithm based on the thermochemical non-equilibrium model of the Martian atmosphere [24]. Finally, aerodynamics and aerothermal prediction algorithms were established to simulate the effects of real gas and rarefaction in a high-temperature Martian atmosphere. Based on comparison of the calculation examples in Fig. 7, it can be confirmed that the current prediction algorithm for the aerodynamic and aerothermodynamic characteristics of the Tianwen-1 entry module is in good agreement with the calculation or flight data of the Viking-1 [25], Mars Pathfinder
(MPF) [26], and Phoenix [27] entry modules. The relative deviation of the aerodynamic force in the rarefied transition flow region is not more than $10 \%$, the relative deviation of the aerodynamic force in the hypersonic continuous flow region is less than $1 \%$, and the relative deviation of the heat flux is not more than $10 \%$.

Figure 8 shows a comparison of the trim drag coefficient and lift-to-drag ratio curves of the Tianwen-1 entry module obtained from the currently established aerodynamic algorithm and those of the Viking-1 entry module obtained using the LAURA code and the flight data [28]. Under the assumption of the same trim angle of attack, the trim drag coefficient and lift-to-drag ratio of the Tianwen-1 entry module are lower than those of the Viking-1 entry module. This is because the afterbody of the Viking-1 entry module is shorter and the inverted cone angle is larger than that of the Tianwen-1 entry

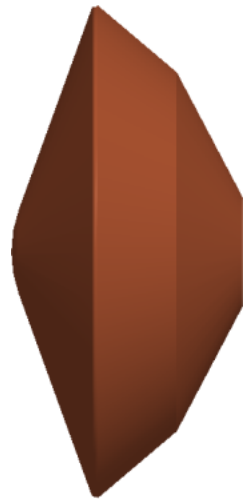

(a) Viking-1 configuration

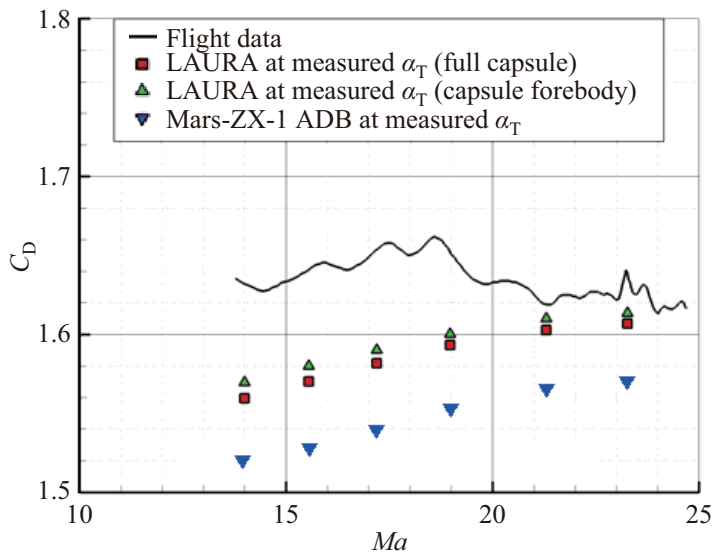

(d) Trim drag coefficient

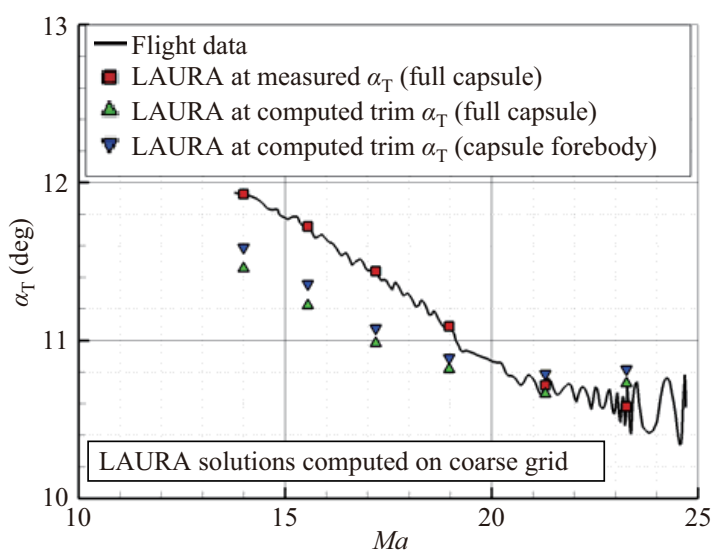

(c) Trim angle of attack

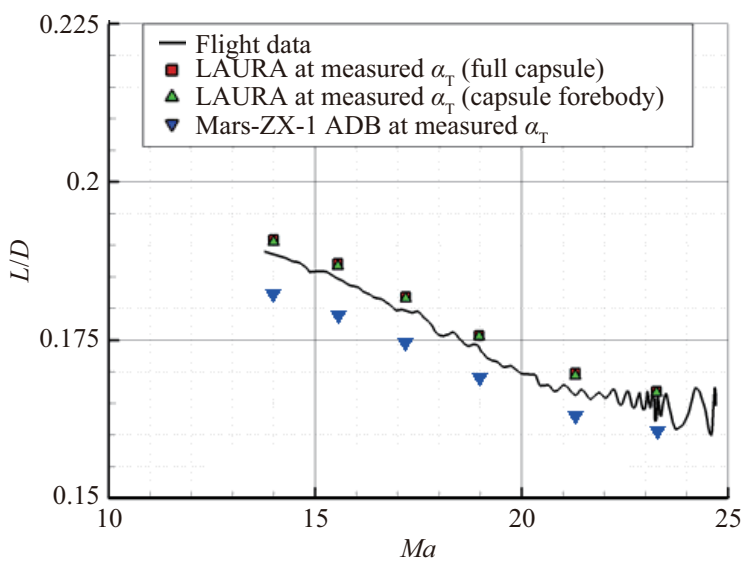

(e) Trim lift-to-drag ratio

Fig. 8 Comparison of the trim aerodynamic characteristics of the Tianwen-1 and Viking-1 entry modules. 
module, and thus the larger separation of the Viking-1 afterbody flow results in a reduction in the afterbody pressure. As a result, the axial force coefficient of the Viking-1 entry module is increased, which improves the drag coefficient and lift-to-drag ratio.

\subsection{Verification techniques for aerodynamic and aerothermodynamic ground tests for Mars entry}

Before the development of Tianwen-1, there were no aerodynamic test facilities in China with $\mathrm{CO}_{2}$ as the main medium. To meet the requirements of the aerodynamic and aerothermodynamic tests and verifications for Tianwen-1, the pneumatic team, in cooperation with domestic pneumatic test sites, carried out technical transformations of the FD-20a gun tunnel and JF-12 high-enthalpy shock wave wind tunnel, established a test flow field for a $\mathrm{CO}_{2}$ medium, and developed a test system

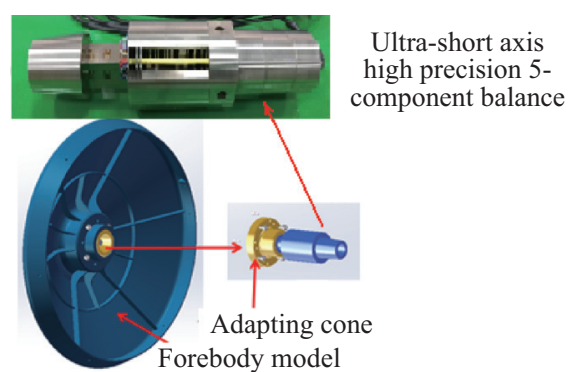

(a) Ultra-short axis high-precision fivecomponent balance for force and heat measurement suitable for the Martian atmosphere medium (including a small interference light piston and diaphragm system, ultra-short axis high-precision five-component balance, and high-precision heat sensor). Based on these facilities and technologies, hypersonic force and heat measurement tests were performed based on the effects of a perfect gas and high-temperature real gas in the air and Martian atmospheric medium, and the influence of the Martian atmospheric medium and its chemical reactions on the aerodynamic and aerothermodynamic characteristics was verified. Figure 9 shows the aerodynamic and aerothermodynamic test equipment for Tianwen-1.

Figure 10 compares the shock fields along the plane of symmetry of the Tianwen-1 entry module using the Mars chemical non-equilibrium numerical calculation and JF-12 wind tunnel tests under typical states of $M a=$ 6.2 and 7.3 and $\alpha=10^{\circ}$. The calculated shock field

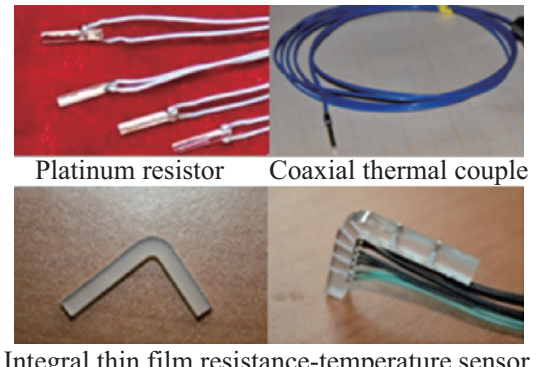

aral thin film resistance-temperature sensor

(b) Heat sensor system with high precision and erosion resistance

Fig. 9 Aerodynamic and aerothermodynamic test equipment for Tianwen-1.

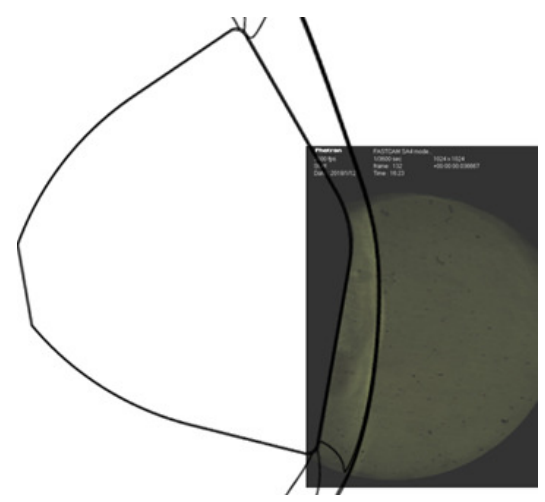

(a) $M a=6.2, \alpha=10^{\circ}$

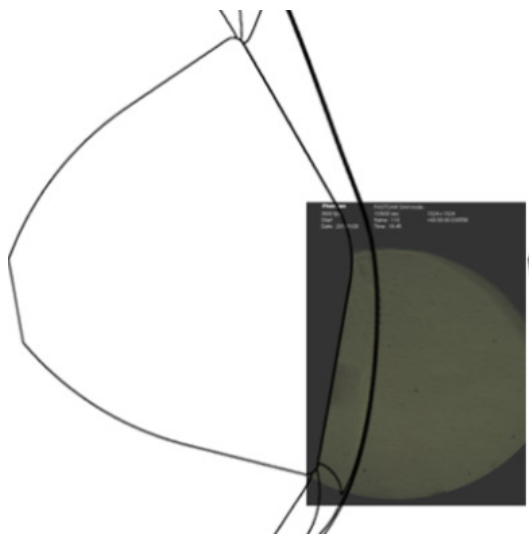

(b) $M a=7.3, \alpha=10^{\circ}$

Fig. 10 Comparison of the shock fields obtained in the JF-12 high-temperature real gas effect experiment and the CFD results (simulated Martian atmosphere). 
morphology is consistent with the experimental results, which verifies the accuracy of the aerodynamic algorithm for the high-temperature real gas effect of the Martian atmosphere.

Figure 11 compares the data curves for the force and heat measurement tests carried out in the FD-20a gun wind tunnel and JF-12 high-enthalpy shock tunnel with air and Mars-like atmospheres, respectively. As seen in Figs. 11(a)-11(c), the entry chamber changes under the influence of both the atmospheric medium and the real gas effect. In detail, the real gas effect of the Martian atmosphere is much more severe, up to $9 \%$, and the axial force of the Martian atmosphere is larger than that of air, with a maximum deviation of approximately $2 \%$. The normal force on the model is basically only affected by the change in medium, with an influence of between $10 \%$ and 20\%. The atmospheric medium of Mars will lead to an increase in the pitching moment of the center of mass, while the real gas effect will lead to a decrease. The combined effect of these two influences will lead to an increase in the absolute value of the trim angle of attack of

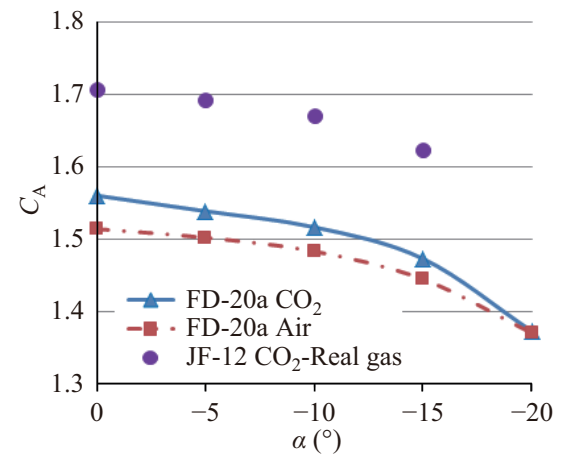

(a) Axial force coefficient

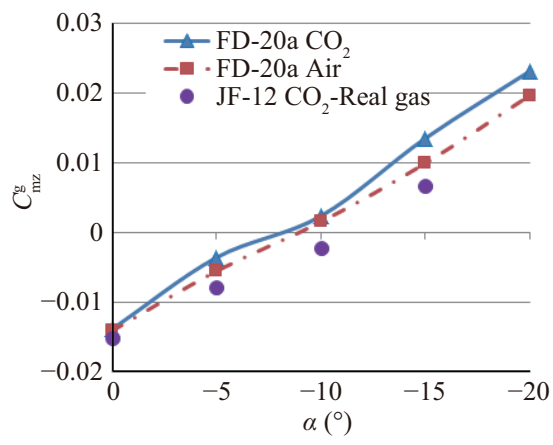

(c) Pitching moment coefficient of the center of mass the entry module. Figure 11(d) shows that the test value of the heat flux on the windward shoulder of the entry module in the Martian atmosphere is lower than that in air, and the peak effect of the shoulder heat flow is not significantly reflected. The heat flux obtained using the aerothermodynamic numerical simulation is very close to the test value for most areas of the forebody but is higher than the test value at the windward shoulder. Therefore, aerothermodynamic design data based on the numerical calculation method are safe for engineering.

\section{Identification of the flight and aerody- namic parameters of Tianwen-1 during the Mars entry}

After Tianwen-1 successfully landed on the surface of Mars, the inertial measurement unit (IMU) data and the surface pressure data of the flush air data system (FADS) were transmitted back to the Earth by the detector's measurement and control data transmission system. Using the obtained pressure distribution data around the center point of the windward head of the

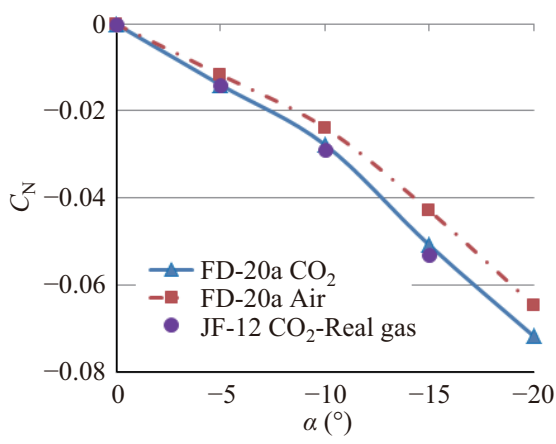

(b) Normal force coefficient

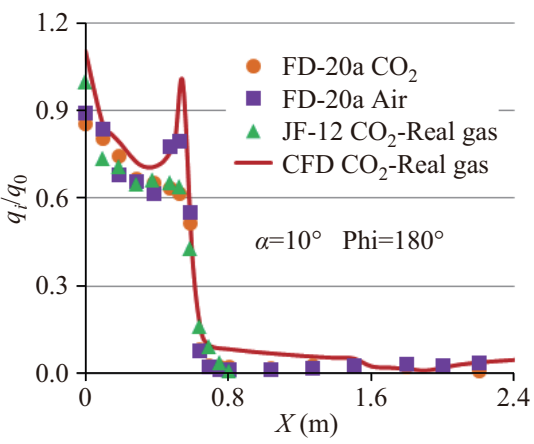

(d) Heat flux on the windward line

Fig. 11 Comparison of the aerodynamic force and heat flux curves in different media obtained in the gun wind tunnel and high-enthalpy shock tunnel $(M a=6.2)$. 
entry module in each state during the entry process, the far-field atmospheric density, atmospheric static pressure, and dynamic pressure data for the entire Tianwen-1 entry process could be obtained using the Kalman filtering method based on the established atmospheric parameter identification model on the ground. Then, based on the six-degree-of-freedom acceleration and attitude angular acceleration data obtained by the IMU, the atmospheric model parameters were substituted into the kinematics and dynamics equations for the entry module and solved simultaneously; thus, the entry trajectory, attitude angle, and velocity of the entry module could be reconstructed, and the aerodynamic parameters of the entry module were identified. Figure 12 compares the changes in the flight parameters, atmospheric parameters, and aerodynamic parameters of the Tianwen-1 entry module along the trajectory obtained using the ground design values and flight values. The design atmospheric parameter data are in good agreement with the flight data in the main aerodynamic deceleration region $(H<40 \mathrm{~km}, t>$ $120 \mathrm{~s}$ ), thus verifying the validity of the design data for the atmospheric parameters. In addition, the deviation between the design value and the flight value of the trim angle of attack of the entry module in the main aerodynamic deceleration region is not more than $1.5^{\circ}$, while the deviation in the trim drag coefficient is not more than $3 \%$. These results indicate that the precision of the aerodynamic calculation and ground test technology adopted for Tianwen-1 is quite high.

It is worth noting that when $H \geqslant 40 \mathrm{~km}$ and $t \leqslant 120 \mathrm{~s}$, the relative measurement error of the FADS pressure sensor leads to inaccurate identification of atmospheric parameters (atmospheric density, pressure, etc.), thus affecting the accuracy of the aerodynamic parameters (drag coefficient, lift coefficient). Therefore, it can be seen in Fig. 12(e) that when $t \leqslant 120 \mathrm{~s}$, the flight value of the drag coefficient for the Tianwen-1 entry module exceeds the predicted error band. However, as shown in Fig. 12(f), the lift-to-drag ratio of the flight data is within the envelope of the design deviation. This is because the influence of the atmospheric model is eliminated when the lift coefficient is divided by the drag coefficient.

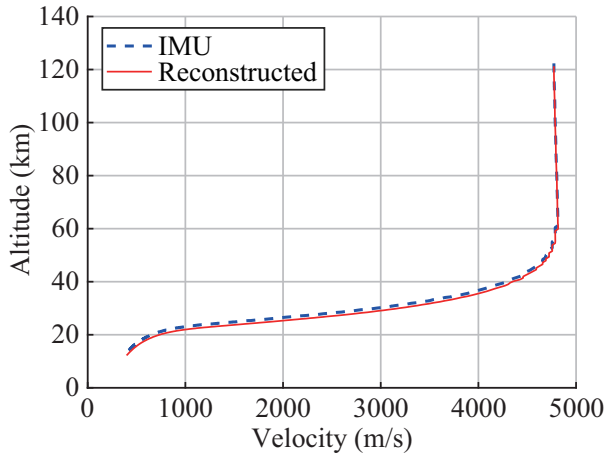

(a) Height vs. velocity

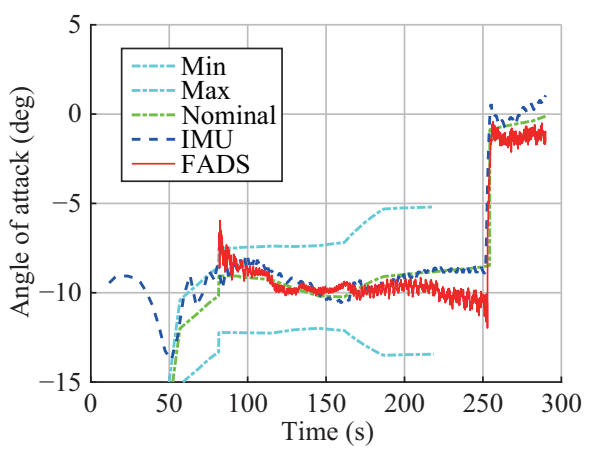

(d) Angle of attack

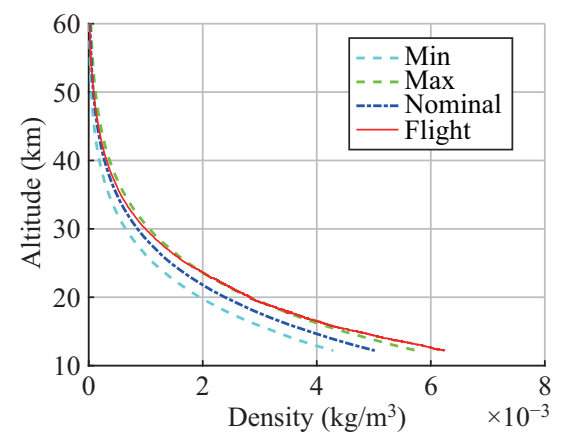

(b) Atmospheric density angle of attack

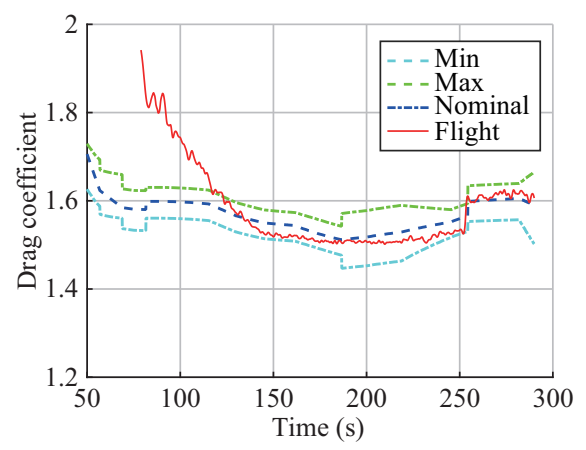

(e) Drag coefficient

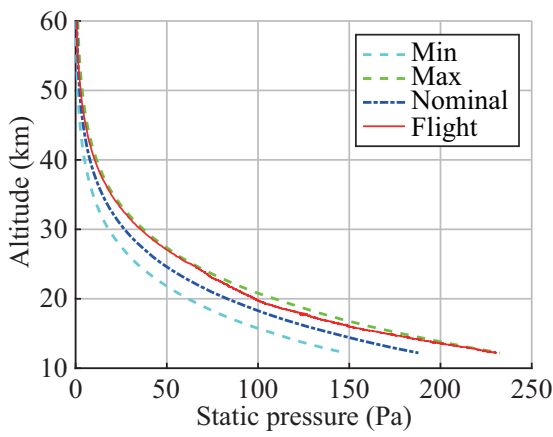

(c) Atmospheric static pressure

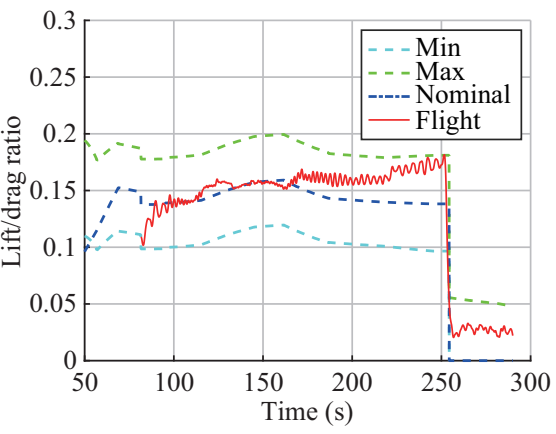

(f) Lift-to-drag ratio

Fig. 12 Comparison of the flight and design results for the flight parameters, atmospheric parameters, and aerodynamic parameters of Tianwen-1 during Mars entry. 


\section{Conclusions}

In the process of the aerodynamic design analysis and verification of Tianwen-1, a series of breakthroughs were made in new technologies, such as the selection and optimization of an aerodynamic shape for high deceleration, aerodynamics/aerothermal calculations, and ground tests based on the Martian atmosphere. During the engineering development, research methods for various complex atmospheric effects were explored, and the level of China's aerodynamic design and research technology for the entry of extraterrestrial bodies has been improved. Comparative analysis reveals that the aerodynamic design data of the Tianwen- 1 entry module are in good agreement with the flight data, which verifies the accuracy of the aerodynamic prediction for Mars entry.

The aerodynamic design, analysis, and verification methods of the Tianwen-1 entry module can not only be applied to EDL missions on Mars but can also be applied to various EDL missions on extraterrestrial bodies with atmospheres, providing significant technical support for the engineering development of new deep-space probes in the future.

\section{Acknowledgements}

This research comes from the Tianwen-1 Mars exploration mission. The authors gratefully acknowledge the contributions of the entire Tianwen-1 design team.

\section{Conflict of interest}

The authors declare that there is no conflict of interest.

\section{References}

[1] Geng, Y., Zhou, J. S., Li, S., Fu, Z. L., Meng, L. Z., Liu, J. J., Wang, H. P. Review of first Mars exploration mission in China. Journal of Deep Space Exploration, 2018, 5(5): 399-405. (in Chinese)

[2] Lyu, J. M., Miao, W. B., Cheng, X. L., Wang, Q. Impact of Martian atmosphere model parameters on aerodynamic characteristics of Mars Science Laboratory. Chinese Journal of Space Science, 2014, 34(4): 377-383. (in Chinese)

[3] Rong, W., Chen, G. L. The characters of deceleration and landing technology on Mars explorer. Spacecraft Recovery E Remote Sensing, 2010, 31(4): 1-6. (in Chinese)

[4] Rao, W., Sun, Z. Z., Meng, L. Z., Wang, C., Ji, L. Analysis and design for the Mars entry, descent and landing mission. Journal of Deep Space Exploration, 2016, 3(2): 121-128. (in Chinese)

[5] Bonn, B. K., Peter, F. I., Alvin, S. Aerodynamic behavior of the Viking entry vehicle ground test and flight results. In: Proceedings of the AIAA 4th Atmospheric Flight Mechanics Conference, 1977.

[6] Edquist, K. T., Dyakonov, A. A., Wright, M., Tang, C. Aerothermodynamic design of the Mars science laboratory heatshield. In: Proceedings of the 41st AIAA Thermophysics Conference, 2009: AIAA 2009-4075.

[7] Willcockson, W. H. Mars pathfinder entry heatshield design and flight experience. Journal of Spacecraft and Rockets, 1999, 36(3): 374-379.

[8] Liang, J., Li, Z. H., Li, Q., Du, B. Numerical simulation of aerodynamic and trim characteristics across different flow regimes for reentry module. Acta Aerodynamica Sinica, 2018, 36(5): 848-855. (in Chinese)

[9] Fang, F., Zhou, L., Li, Z. H. A comprehensive analysis of aerodynamics for spacecraft re-entery Earth's atmosphere surroundings. Acta Aeronautica et Astronautica Sinica, 2015, 36(1): 24-38. (in Chinese)

[10] Li, Q., Wei, H. G., Li, Z. H., Chen, G., Peng, J., Yang, M. F. Prediction and flight verification of chemical non-equilibrium aerodynamic characteristics of the high-speed circumlunar return and reentry capsule. SCIENTIA SINICA Technologica, 2015, 51(7): 813-827. (in Chinese)

[11] Lyu, J. M., Cheng, X. L., Wang, Q. Flow field prediction of an orbiter entering the Mars atmosphere. Chinese Journal of Space Science, 2013, 33(2): 129-134. (in Chinese)

[12] Cheng, X. L., Li, J. H., Wang, Q. Aerodynamic force characteristics of Mars entry vehicles. Journal of Astronautics, 2010, 31(4): 967-972. (in Chinese)

[13] Wang, R., Chen, B. Y. Aerodynamic configuration design optimization of reentry capsule based on CFD and multi-objective optimization theory. Acta Aerodynamica Sinica, 2015, 33(5): 588-593, 609. (in Chinese)

[14] Braun, R. D., Manning, R. M. Mars exploration entry, descent, and landing challenges. Journal of Spacecraft and Rockets, 2007, 44(2): 310-323.

[15] Liu, Z. Y., Li, Q., Wei, H. G., Geng, Y. F. Performance comparison of typical semi-ballistic Mars entry vehicles. Acta Aerodynamica Sinica, 2018, 36(5): 863-870. (in Chinese)

[16] Yang, X. F., Tang, W., Gui, Y. W., Xiao, G. M., Feng, Y. Study on aerodynamic configuration of Mars probe. Manned Spaceflight, 2015, 21(4): 412-417. (in Chinese)

[17] Kipp, D., Martin, M. S., Essmiller, J., Way, D. Mars Science Laboratory entry, descent, and landing triggers. 
In: Proceedings of the IEEE Aerospace Conference, 2007: $1-10$.

[18] Edquist, K., Desai, P., Schoenenberger, M. Aerodynamics for the Mars phoenix entry capsule. In: Proceedings of the AIAA/AAS Astrodynamics Specialist Conference and Exhibit, 2008: AIAA 2008-7219.

[19] Tang, W., Yang, X. F., Gui, Y. W., Du, Y. X. Review of hypersonic aerodynamics and aerothermodynamics for Mars entries. Journal of Astronautics, 2017, 38(3): 230-239. (in Chinese)

[20] Lyu, J. M., Miao, W. B., Huang, F., Cheng, X. L. Model analysis for predicting aerodynamic characteristics of Mars entry. Chinese Journal of Space Science, 2016, 36(3): 344-351. (in Chinese)

[21] Lyu, J. M., Miao, W. B., Huang, F., Cheng, X. L. Effect of high temperature gas in shock layer on aerodynamic characteristics of Mars entry vehicle. In: Proceedings of the China Mechanics Congress, 2015.

[22] Liu, Q. Z., Dong, W. Z., Ding, M. S., Jiang, T., Gao, T. S. Numerical simulation of aerothermal environments and aerodynamic characteristics for Mars entry capsules. Acta Aerodynamica Sinica, 2018, 36(4): 642-650. (in Chinese)

[23] Miao, W. B., Lyu, J. M., Cheng, X. L., Ai, B. C. Numerical analysis of thermodynamics models for Mars entry aeroheating prediction. Chinese Journal of Computational Physics, 2015, 32(4): 410-415. (in Chinese)

[24] Huang, F., Lyu, J. M., Cheng, X. L., Li, Q. Aerodynamics of Mars entry vehicles under hypersonic rarefied condition. Acta Aeronautica et Astronautica Sinica, 2017, 38(5): 120457. (in Chinese)

[25] Wilmoth, R. G., Blanchard, R. C., Moss, J. N. Rarefied transitional bridging of blunt body aerodynamics. In: Proceedings of the 21st International Symposium on Rarefied Gas Dynamics, 1998.

[26] Bose, D., Wright, M. Uncertainty analysis of laminar aeroheating predictions for Mars entries. In: Proceeding of the 38th AIAA Thermophysics Conference, 2005: AIAA 2005-4682.

[27] Edquist, K., Desai, P., Schoenenberger, M. Aerodynamics for the Mars phoenix entry capsule. In: Proceedings of the AIAA/AAS Astrodynamics Specialist Conference and Exhibit, 2008: AIAA 2008-7219.

[28] Karl, T. E. Computations of Viking lander capsule hypersonic aerodynamics with comparisons to ground and flight data. In: Proceedings of the AIAA Atmospheric Flight Mechanics Conference and Exhibit, 2006: AIAA 2006-6137.

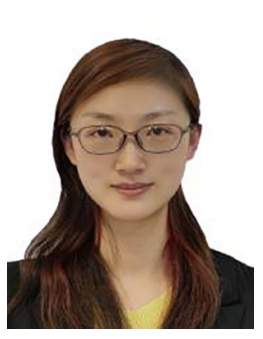

Qi Li was graduated from Beihang University in 2008 with a major in fluid mechanics. As the head of the entry and re-entry pneumatic department, she is responsible for the aerodynamic design, analysis and verification of Chang'e- 5 and Mars probes. She has won the first prize of Science and Technology Progress of Sichuan Province and the second prize of Science and Technology Progress Award of China Aerospace Science and Technology Corporation.

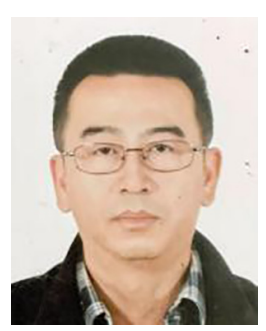

Wei Rao was graduated from Beihang University in 1993. He participated in the development of Chang'e-1, Chang'e-2 lunar probes and Mars probes, serving as the product assurance manager and deputy designer of the Mars probes. He has won the third prize of National Defense Science and Technology Progress Award once, the special prize of National Science and Technology Progress Award twice, and the National Outstanding Youth, etc.

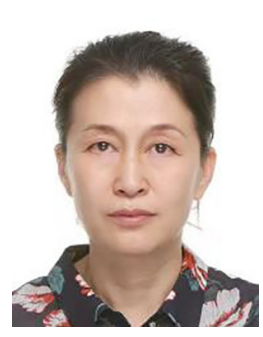

Xiaoli Cheng has long been engaged in the research of high-temperature real gas effect and rarefied effect.

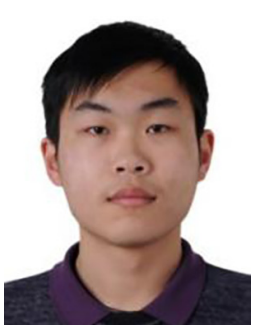

Haogong Wei achieved his M.S. degree at Purdue University in 2014. Since then he participated in China's deep space exploration programs at Beijing Institute of Spacecraft System Engineering, focusing on flight dynamics and aerodynamics.

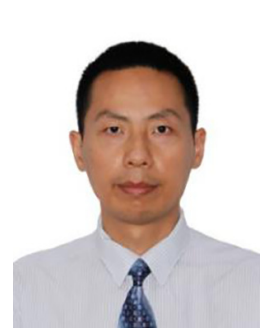

Chuang Wang joined China Academy of Space Technology (CAST) in 2008. His research filed is overall design of deep space probe. He has won one third prize of Science and Technology Progress Award of China Aerospace Science and Technology Corporation and one third prize of Science and Technology Progress Award of Beijing. 
Open Access This article is licensed under a Creative Commons Attribution 4.0 International License, which permits use, sharing, adaptation, distribution and reproduction in any medium or format, as long as you give appropriate credit to the original author(s) and the source, provide a link to the Creative Commons licence, and indicate if changes were made.

The images or other third party material in this article are included in the article's Creative Commons licence, unless indicated otherwise in a credit line to the material. If material is not included in the article's Creative Commons licence and your intended use is not permitted by statutory regulation or exceeds the permitted use, you will need to obtain permission directly from the copyright holder.

To view a copy of this licence, visit http://creativecomm ons.org/licenses/by/4.0/. 